Cahiers d'ethnomusicologie

\title{
Cinuçen tanrikorur et la tradition du ud turc
}

\section{Nur Dervis}

Traducteur : Nur Dervis

\section{OpenEdition}

\section{Journals}

Édition électronique

URL : http://journals.openedition.org/ethnomusicologie/2349

ISSN : 2235-7688

\section{Éditeur}

ADEM - Ateliers d'ethnomusicologie

\section{Édition imprimée}

Date de publication : 1 janvier 1989

Pagination : 255-262

ISBN : 2-8257-0178-5

ISSN : $1662-372 X$

\section{Référence électronique}

Nur Dervis, "Cinuçen tanrikorur et la tradition du ud turc », Cahiers d'ethnomusicologie [En ligne],

2 | 1989, mis en ligne le 15 septembre 2011, consulté le 01 mai 2019. URL : http://

journals.openedition.org/ethnomusicologie/2349 


\section{CINUÇEN TANRIKORUR et la tradition du ud turc}

Cinuçen Tanrıkorur est né à Istanbul en 1938. Baigné dans l'ambiance de la musique traditionnelle, il se consacre à cet art dès son plus jeune âge. C'est en autodidacte qu'il aborde le chant, le jeu du luth $(u d)$ et la composition. Exception qui confirme la règle, il ne suit pas le long cheminement du contact régulier avec un maître exclusif. Ses dons hors pair lui permettent de décrypter et d'assimiler les arcanes de l'art - règles de chaque mode musical (makam), dynamique de chaque cycle de composition (usûl) - et surtout de systématiser l'édifice musical classique turc.

Après des études secondaires au Lycée italien, il obtient son diplôme d'architecture à l'Académie des beaux-arts. C'est cependant à la construction d'édifices sonores que son talent allait s'exercer. A vingt-deux ans, il devient musicien à Radio Istanbul; il s'attaque ensuite à la rédaction d'une méthode d'enseignement du luth, et devient responsable de la section de musique traditionnelle à Radio Ankara. Il est de plus aujourd'hui professeur de musicologie à l'Université de Konya.

Compositeur de talent, Cinuçen est l'auteur de plus de cent pièces, tant vocales qu'instrumentales, ainsi que le créateur d'un nouveau mode, le makam Seddisabâ, qu'il expose dans une suite classique (fasll) en six mouvements. Il est aujourd'hui le seul musicien classique ayant renoué avec la tradition des ozan, les troubadours turcs d'Asie, le seul à interpréter les compositions savantes, du XVI ${ }^{\mathrm{e}}$ siècle à nos jours, sur l'unique accompagnement de son luth.

Inspiré par l'héritage ancestral qu'il vitalise, Cinuçen Tanrıkorur cristallise les transports de son imagination dans les formes traditionnelles de composition (peşrev, semaï, beste, etc.) et d'improvisation (taksim). Son style a été décrit comme une sorte de paroxysme de l'art turc savant. Sa voix se caractérise par la variété et la subtilité des intonations et des timbres, et son jeu instrumental par une expression formelle inhabituelle d'un fond intégralement traditionnel. 
Nur Derviş : Je crois que vous avez récemment soumis au Ministère turc de l'éducation nationale un projet de modification de l'enseignement musical dans les écoles secondaires. En quoi consiste-t-il?

Cinuçen Tanrıkorur: Nous envisageons une répartition plus équitable de la musique occidentale et de la musique turque. La proportion actuelle est de $97,5 \%$ de musique occidentale pour $2,5 \%$ de musique turque. Il ne s'agit bien sûr pas de l'inverser, mais de viser à ce qu'elle soit de $50 \%$ pour chacune. Compte tenu de la situation géographique de la Turquie, il est indispensable d'équilibrer la balance. Un musicien turc doit aujourd'hui absolument connaître les deux systèmes musicaux pour qu'un réel renouvellement de notre musique puisse avoir lieu. La solution n'est pas, à mon avis, d'effectuer des emprunts comme ceux tentés par des compositeurs comme Adnan Saygun et ses amis avec leur «Quintette turc» inspiré du Quintette russe, mais de réaliser une musique turque contemporaine ayant une base classique et un contenu contemporain, avec des makam ${ }^{1}$ (modes mélodiques) turcs, des usul (cycles rythmiques) turcs, des instruments de musique fabriqués selon les canons esthétiques et acoustiques turcs.

\section{N.D.: Pensez-vous qu'une telle musique puisse intéresser les Occidentaux?}

C.T.: J'ai personnellement quelques amis occidentaux qui s'intéressent à la musique turque. Certains sont mes élèves, et je leur dis la même chose: pour aborder la musique turque, il leur faut d'abord bien connaître leur propre musique. A priori, l'homme de la rue occidental ne s'intéresse pas à la musique turque parce qu'elle ne correspond pour lui à aucun vécu, et qu'il ne s'intéresse de toute façon pas de près à sa propre musique. Mais pour un Occidental sensible à la musique et dépourvu de préjugés, la musique turque est susceptible d'éveiller un grand intérêt.

Parmi les musiciens turcs, il subsiste aujourd'hui une bonne dose de complexe d'infériorité, et je crains que cela dure encore un certain temps. Ils ont l'impression que les Occidentaux perçoivent la musique turque comme une curiosité exotique, au même titre que la musique cambodgienne ou malgache. Ils imaginent par exemple que, quand je vais en Europe, je joue pour les ouvriers turcs émigrés. Ce sentiment est évidemment conditionnél par leur environnement. Depuis le tanzimat ${ }^{2}$ - qui a mon sens a été un véritable tremblement de terre - la plupart des Turcs, et en particulier des intellectuels, ont été atteints d'un grave traumatisme en ce qui concerne l'opposition «alla turca - alla franca». Ainsi, tout ce qui vient de l'Occident, y compris le pire, leur paraît beau, et toutes nos valeurs leur semblent laides!

Nous avons respecté l'orthographe actuelle des mots turcs. Par contre, la transcription des termes arabes suit l'usage académique courant.

2 Tanzimat est le nom de la réforme juridique et administrative de l'Empire ottoman, entreprise en 1839 et achevée vers 1890 . Cette réforme avait pour but de mettre la Turquie sur la voir de l'occidentalisation. 


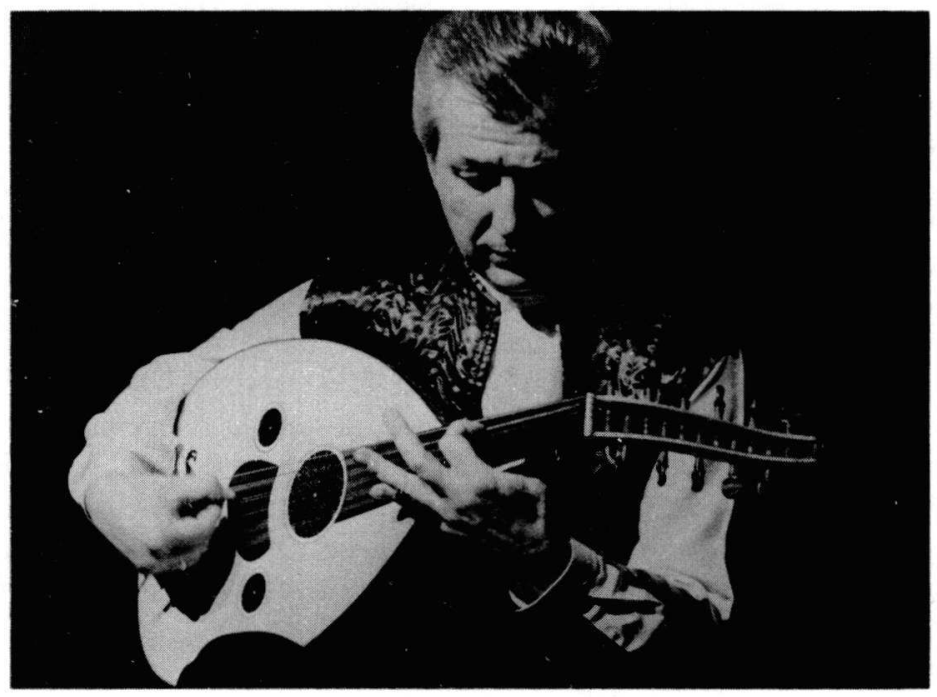

N.D.: Ce que vous dites est tout à fait vrai. D'un côté, on idéalise l'Occident et de l'autre, on se dévalorise en se voyant avec un regard d'Occidental. En réalité, cela est dû à une méconnaissance de l'Occident.

C.T.: Si nous avions la possibilité de mieux le connaître, toutes ces erreurs ne se seraient évidemment pas produites. Nous aurions pu savoir ce qui était bon à prendre et ce qu'il fallait à tout prix éviter. Dans le domaine musical, la confrontation avec l'Occident permet de mettre en évidence le critère de l'authenticité. En général, les Occidentaux s'intéressent à la musique turque, ou de quelque autre pays, dans la mesure où elle est authentique et traditionnelle. Plus d'un musicien traditionnel oriental est ainsi adulé en Europe et totalement ignoré dans son propre pays.

\section{N.D.: Pourriez-vous nous dire quelques mots sur le passé de la musique tur- que?}

C.T.: Ses premières traces connues remontent aux Huns, qui utilisaient la musique notamment à des fins militaires. On pense toujours que le mehter (l'orchestre des janissaires de l'armée ottomane) est né sous les Ottomans. Or ils l'ont hérité des Huns, et il était aussi connu des Götzürk, des Uygur, des Karahanl et des Selçuk (Seldjoukides). Le mehter était à l'origine constitué de cinq instruments, dont deux instruments à vent et trois percussions, que les Huns appelaient yurag, borguy, tümrük, küvrük et çang. Après l'islamisation des Turcs, le yurag est devenu le zurna (surnay en persan), le borguy s'est appelé boru, "tuyau» (nevchir en persan), le küvrük est aujourd'hui connu sous le nom de $k o ̈ s ;$ le nom de çang est une onomatopée, alors que le tümrük est devenu le tambour que nous appelons aujourd'hui davul. Ce nom vient de l'arabe al$t a b l$, qui dérive lui-même soit du latin tavola, soit du sanscrit tap. 
Cet ensemble musical comporte encore le bâton que tient le chef du groupe pour marquer le rythme en marchant devant les autres musiciens; en langue hun, il s'appelle çokan ou çöven, ce qui a donné en persan çevgan. Les Anglais l'ont par la suite emprunté et lui ont donné le nom de joanna, puis de jingling johnny, qu'ils désignent comme un "croissant turc» (Turkish crescent). Cet instrument est orné de clochettes, de rubans et de certains signes symboliques qui attestent encore son origine chamanique. Le chamane était à la fois prêtre, sorcier, guérisseur, dentiste, barbier, musicien, poète, et il utilisait cet instrument comme bâton de rythme dans la pratique de certains rites.

Un autre instrument ancien, qui n'a rien à voir avec la musique militaire, est le luth kopuz. Le Dedekorkut, une saga turkmène d'Asie centrale, le mentionne sous le nom de kolca kopuz, kopuz «à bras». Le nom du colascione italien dérive de kolca kopuz. Quant à l'instrument, il est probablement un ancêtre de l'actuel $s a z$, le luth à long manche de la musique populaire que les ouvriers turcs émigrés en Allemagne emportent souvent avec eux, et qui est pour eux l'emblème de leur rattachement à la patrie.

Parmi les luths à long manche, il faut aussi mentionner le tanbur, que les Arabes appellent parfois tanbūr al-kabìr al-turqī, le «grand tanbūr turc», qui a joué un rôle important dans la théorie de la musique turque. La disposition des frettes sur son manche permet en effet de comprendre le système mélodique turc. Vous n'avez pas la même chose sur le $u d$, qui n'a pas de frettes. Le tanbur a huit cordes réparties en quatre chœurs, et ses mélodies se jouent "verticalement» sur le premier chœur, en déplaçant la main gauche de haut en bas du manche. Par contre, le jeu du ud est essentiellement «latéral», c'est-à-dire que les mélodies sont produites en passant d'un chœur à l'autre.

\section{N.D.: De tout ce que vous dites, il ressort que la musique turque s'est consi- dérablement modifiée avec le temps. Quelles sont les tendances marquantes de ces transformations?}

C.T.: Il me faut commencer par rappeler deux caractéristiques fondamentales de la musique classique turque: c'est une musique de chambre, et qui est essentiellement vocale. C'est pourquoi le fait de la chanter avec des chœurs de quarante à cinquante personnes, comme cela se fait souvent à la radio, est ridicule. C'est un emprunt aux grands chœurs de la musique symphonique occidentale, eux-mêmes dérivés de ceux de la Grèce antique. Dans les longes suites classiques ( $\mathrm{fasl} l$ ), l'introduction du chœur et le grossissement de l'orchestre ont complètement dénaturé cette musique. Sous l'influence occidentale, l'aspect quantitatif des choses a pris plus d'importance, et on a cru bien faire en appliquant ce principe à la musique. Après avoir été un art de chambre, elle est devenue un art de salon, puis de scène, avec l'introduction de cette dimension symphonique. Et pourtant, dans le passé de la musique turque, le chœur est inexistant. Tout y est basé sur une voix soliste, éventuellement accornpagnée d'un ou deux instruments. C'est dans cette grande simplicité que l'expression musicale turque a atteint ses sommets. 


\section{N.D.: En va-t-il de même en ce qui concerne la musique populaire?}

C.T.: Jadis, la poésie populaire était interprétée par les bardes qu'on appelait les ozan. Lorsqu'un ozan arrivait dans un village et se mettait à chanter, tout le monde arrêtait son travail et se taisait pour l'écouter. Dès le $\mathrm{XV}^{\mathrm{e}}$ siècle, la tradition des ozan a été perpétuée par les aşık (litt.: "amoureux», troubadours), et nous avons encore aujourd'hui d'excellents aşık. Un des grands chanteurs et joueurs actuels de bağlama (variété de saz) est Orhan Gencebay. C'est un musicien extraordinaire, dont la musique peut vous faire frisonner; cela dépasse la compréhension. A mon avis, il est le plus grand musicien turc contemporain, bien qu'il soit souvent critiqué jusque dans les milieux cultivés sous prétexte qu'il abuse de l'arabesque. On raconte qu'il est alcoolique, toxicomane, ou je ne sais quoi ... Le pauvre, alors qu'il ne boit que de l'eau et qu'il ne fume même pas de cígarettes! C'est aussi un remarquable poète, qui s'inspire de chef-d'œuvres, tels que le Mesnevî de Rûmî ou le Gülistan de Sâdi. C'est un être lumineux, plein de talent et qui connaît bien sûr parfaitement la musique. Que Dieu le garde!

N.D.: Et pourtant, il introduit des éléments électroniques dans sa musique. Cela ne vous gêne pas?

C.T.: Je trouve cela tout à fait normal. Orhan n'est pas un musicien classique; il fait de la musique populaire actuelle, et c'est un domaine dans lequel il n'y a pas de contraintes, pas de limitations. La seule question qui se pose est de savoir si ce qu'il fait est beau, s'il a un but, un message à transmettre, et si sa musique touche le public. Il est clair que, selon nos critères d'intellectuels, ce n'est pas cela qui entre en ligne de compte. Mais il est trop facile de le rejeter sous prétexte qu'il représente par exemple une tendance décadente. A mon avis, dans ce domaine, ce sont les goûts du peuple qui sont déterminants. Les Turcs sont trop musiciens pour se laisser séduire par n'importe quoi.

N.D.: J'aimerais maintenant que vous parliez de votre instrument, le ud, et de son rapport avec la culture et la spiritualité islamiques. On sait par exemple que, en parlant du ney (flûte de roseau), Mevlâna Celaleddin-i Rûmî dit que le son de cet instrument évoque les soupirs et les lamentations du roseau coupé de ses racines, séché, vidé et percé de trous. Trouve-t-on une telle vision mystique de l'instrument à propos du ud?

C.T.: Personnellement, je ne connais pas d'interprétation semblable concernant le $u d$. Ce que je peux vous dire serait limité à des considérations historiques. Le $u d$ est généralement considéré comme un instrument arabe implanté dans la culture musicale turque. La plupart des Turcs répugnent à le voir comme un instrument turc à part entière, ce qui est absurde, car on connaît son ancienneté dans notre musique.

L'islamisation de la Turquie remonte au VIII ${ }^{\mathrm{e}}$ siècle et elle s'est stabilisée deux siècles plus tard, du temps de Sarı Saltuk. C'est à cette époque que des émigrés du Khorasan venus de Baghdad ont apporté le $u d$, dont le nom vient de 
l'arabe $a l$-'ūd, comme d'ailleurs les noms européens du luth - liuto en italien, laud en espagnol, alaud en portugais, lute en anglais, Laute en allemand, etc. Mais au début, les Turcs appelaient cet instrument kopuz, puis son nouveau nom s'est imposé parce qu'il était fait en bois d'aloès, al-'úd en arabe. Il avait alors chez nous des cordes en soie, dont les Turcs avaient appris la facture des Chinois. Les annales chinoises attestent en effet que, notamment sous la dynastie des Tchou, les maîtres de musique de la cour impériale étaient des Huns. Ce n'est que l'impérialisme culturel européen qui a représenté les Huns comme des barbares rustres et sanguinaires. Mais c'était un peuple très raffiné: pour eux comme pour les Ottomans, il n'aurait pas été possible de fonder et de maintenir un tel empire uniquement par le sabre.

On pourrait faire des remarques semblables sur la cithare kanun. Ce mot vient de l'arabe qanūn, lui-même dérivé du grec kanon, «règle». Les Huns, qui connaissaient cet instrument, l'appelaient yatuk ou yatağan, de la racine YAT, «coucher», parce qu'on le "couche» sur ses genoux. Un autre dérivé du yatuk est le santūr, un des principaux instruments de la musique d'Iran et du Cachemire. Sa principale différence avec le kanun réside dans le fait que ses cordes sont frappées à l'aide de baguettes, et non pincées par des onglets comme celles du kanun.

Pour en revenir au $u d$, cet instrument a connu une période de déclin dans le sérail ottoman jusqu'au XVIII ${ }^{\mathrm{e}}, \mathrm{XIX}^{\mathrm{e}}$ siècle. A cette époque, le violoncelliste Şakir Paşa a réintroduit le $u d$, suivi par son élève Şerif Muhiddin et ses contemporains Udî Nevres Bey, Şerif Içli et Yorgo Bacanos, qui furent tous de véritables maîtres du $u d$.

\section{N.D.: Quelles sont les circonstances qui vous ont poussé à aborder le ud plutôt qu'un autre instrument?}

C.T.: Ma mère jouait déjà du $u d$ depuis toute jeune. J'ai donc été bercé au son du $u d$, mais dans mon enfance, je n'étais pas particulièrement attiré par cet instrument. Par contre, je chantais, je battais les mesures du fasll dans mes mains, et dès l'âge de huit ans, j'ai commencé à apprendre à lire la musique; mon père avait acheté un petit recueil de chansons d'Iskander Kutmani, où la musique était notée avec les paroles. Comme je connaissais les chansons, j'ai ainsi pu en déchiffrer la notation musicale.

Lorsque j'ai perdu ma mère en 1956, j'ai eu son $u d$ entre les mains pour la première fois de ma vie. Ce n'était pas un choix délibéré, et si ma mère avait joué du kanun ou du tanbur, j'aurais joué de cet instrument.

\section{N.D.: Vous avez donc appris la musique sans maître?}

C.T.: Oui, Dieu merci! Je crois d'ailleurs que la présence d'un maître ne m'aurait pas convenu. Cela ne veut évidemment pas dire que je n'ai rien appris de qui que ce soit; au contraire, j'écoutais très attentivement tous les musiciens qu'il m'était donné d'entendre. A cette époque, mon idole était le grand udiste Yorgo Bacanos. Dès que j'entendais un de ses taksim à la radio, je m'empressais de l'imiter. Je pouvais presque visualiser son doigté. Je fermais les yeux 
et m'efforçais d'imaginer comment il réalisait telle ou telle mélodie. J'essayais ensuite de la reproduire sur mon $u d$ jusque dans ses moindres détails.

En 1960, quatre ans après mes débuts, je me suis présenté au concours de musique de Radio Istanbul. J'étais inscrit pour le chant et le $u d$; et, bien que je me sois senti plus sûr de ma voix que de mes qualités instrumentales, j'ai gagné le concours de $u d$. Mais ma technique était loin d'être parfaite. Cinq ans plus tard, j'ai entendu pour la première fois le grand joueur de tanbur Tanburî Cemil, et son jeu m'a bouleversé. J'ai par la suite été à ses côtés les cinq dernières années de sa vie. J'étais complètement fasciné par son style et sa technique de tanbur, que je me suis efforcé d'adapter au $u d$. Mais il serait présomptueux de prétendre que j'y suis arrivé. Certains de mes collègues comme Necdet Yaşar et Ihsan Özgen ont aussi été très proches de Cemil. Et bien qu'il soit avant tout connu comme un virtuose du kemençe, Ihsan est à mon avis avant tout un grand joueur de tanbur.

Pour moi, le grand enseignement de Cemil, sur le plan technique, a été celui du jeu «vertical» dont je parlais tout à l'heure. Cette manière de jouer présente le grand intérêt d'être plus proche des inflexions de la voix. Je l'ai intégré à tel point que certaines personnes m'appellent aujourd'hui udburî! Mais cela m'a aussi valu des critiques, On prétend qu'en m'écoutant, il est impossible de savoir si je joue de la guitare, du baǵlama ou du bouzouki. D'autres disent que, grâce à cette adaptation, j'ai permis de hisser le $u d$ turc au niveau des instruments de récital, que je lui aurais rendu sa noblesse. Mais tous ces commentaires ne me préoccupent pas beaucoup.

\section{N.D.: Vous avez longtemps travaillé pour la radio-télévision turque. Que retirez-vous de cette expérience?}

C.T.: Un souvenir très pénible! Travailler à la radio-télévision turque comme musicien est pire que d'être condamné à quarante ans de prison! En vingt-cinq ans de service, j'ai bien failli être dégoûté à jamais de la musique. J'étais entouré de gens ayant une mentalité de fonctionnaire et dont l'intérêt pour la musique cessait dès l'instant où ils sortaient du studio d'enregistrement. Il m'a été bien difficile d'y préserver mon esprit d'amateur passionné. Une fois mes obligations quotidiennes terminées, je restais fréquemment des heures seul en studio à travailler mon $u d$, car je savais que, dès qu'on écarte l'instrument de son corps, il se réfroidit et, de ce fait, perd son accord, il ne répond plus.

\section{N.D.: L'instrument est donc comme un être vivant?}

C.T.: Bien sûr, il est pour moi le meilleur des amis, un compagnon inséparable. D'ailleurs, vous avez vu: je le tiens entre mes bras comme un bébé, et lorsque j'arrête de jouer, je le pose face à mes genoux, pour préserver sa température. Le rapport à un instrument de musique est comme une relation humaine, qui se développe ou décline en fonction de l'attention qu'on y porte. Dieu merci, nous nous aimons, mon $u d$ et moi! 


\section{N.D.: Le ud est-il aujourd'hui un instrument populaire en Turquie?}

C.T.: Il est curieux de constater que, depuis une dizaine d'années, le $u d$ semble être l'instrument le plus vendu en Turquie, au point que les luthiers n'arrivent pas à répondre à la demande. Le point positif est que cela crée de nouveaux emplois dans cette branche, et je serais très honoré de penser que j'y suis peut-être pour quelque chose. Il existe bien sûr une très grande différence qualitative entre les grands $u d$ des anciens luthiers et la plus grande partie de la production actuelle. Mais le fait que de jeunes apprentis toujours plus nombreux se tournent vers cet artisanat est très encourageant pour l'avenir du $u d$, inch'Allâh!

Propos recueillis et traduits du turc* par Nur Derviş

\section{Discographie de Cinuçen Tanrıkorur}

Hormis d'innombrables enregistrements réalisés pour la radio turque, dont certains ont fait l'objet de cassettes pirates vendues en Turquie, la discographie «officielle», accessible en Occident, de Cinuçen Tanrıkorur se résume actuellement aux deux disques suivants:

1971 Musique traditionnelle turque. Paris: OCORA OCR 56.

A2: Solo de ud (taksim - makam Segâh)

A3: Ensemble instrumental (peşrev - makam Segâh)

B1: Ensemble instrumental (sazkâr semaï - makam Sazkâr)

B2: Gerdaniye Peshrev (makam Gerdaniye)

B3: Aksak Semaï (makam Gerdaniye)

1986 Turquie. Cinuçen Tanrıkorur. Paris: OCORA OCR 558574.

A1: Bayatîaraban Ayîn-î Şerîfi

B1: Taksim Bestenigâr

B2: Elégie à Akagündüz Kutbay (makam Bestenigâr)

B3: Taksim Hüseyni

B4: Le retour des champs (aksak semaï, yürük semaï - makam Hüseyni).

* La traduction a été partiellement remaniée par Laurent Aubert. 